Bull. Egypt. Soc. Physiol. Sci. 40 (2), 154-164

\author{
Bull. of Egyp. Soc. Physiol. Sci. \\ (Official Journal of Egyptian Society for Physiological Sciences) \\ (pISSN: 1110-0842; eISSN: 2356-9514)
}

\title{
Is There a Possible Impact of Erythropoietin Hormone on Peripheral Neuropathy going with Acute Renal Damage in Fully-Grown Male Rats?
}

\author{
Fatma Hassan $^{1}$, Dalia Mahmoud Eid ${ }^{2}$ \\ ${ }^{1}$ Physiology Department, Faculty of Medicine, Cairo University, Egypt \\ ${ }^{2}$ Biochemistry Department, Faculty of Medicine, Ain Shams University, Egypt
}

Received: 8 March 2020

Accepted: 18 May 2020

Available online: 1 July 2020

\section{Keywords}

- Acute renal failure

- Amplitude

- Erythropoietin

- Nerve conduction velocity

- Nerve growth factor

- Neuropathy

- Oxidative stress

\begin{abstract}
Background: erythropoietin hormone (EPO) with its great built erythropoietic impact, has recorded a lot of protective impacts on particular body organs and tissues. A standout amongst these is its renoprotective impact which is interceded through a various mechanisms. Objective: as neuropathy had been reported frequently amongst patients with severe renal damage, this work had been made to record any possible benefits of EPO on the peripheral neuropathy that might go with acute renal failure (ARF). Methods: this study was carried out in Faculty of Medicine, Cairo university, in January2019 , on forty rats that had been randomly separated into four groups; Group I: control rats, Group II: ARF, Group III: ARF+EPO, and Group IV: EPO+ARF. Results: EPO injection had significantly reduced the levels of serum creatinine, blood urea nitrogen and (malondialdehyde and nerve growth factor within the nerve fibers). Also 24-hours urine volume, glomerular filtration rate, nerve conduction velocity and amplitude besides neural level of superoxide dismutase had been fundamentally improved. Conclusion: from this study we had concluded that EPO intake had exerted protective impacts on peripheral neuropathy following ARF.
\end{abstract}




\section{INTRODUCTION}

Acute renal failure (ARF) represents abrupt tremendous drop of kidney functions particularly glomerular filtration rate (GFR), resulting in accretion of various harmful metabolites within hours or days (1). A standout amongst these unsafe metabolites is urea causing uremia that provoke multiorgan dysfunction, and tissues damage yielding a high mortality despite the availability of different medications (1). A standout amongst those tissues that could be influenced by the intense kidney damage- with markedly decreased GFR - is the peripheral nerves (2).

Uremic neuropathy that is usually reported in cases of sudden demise extreme renal shutdown is of a gradual onset and progressively deteriorating course which basically influences the thick myelinated nerves. It might be accidently discovered. The earliest and commonest symptoms and signs are parasthesia and loss of vibration sense respectively. There might be autonomic debilitation (3). But, hopefully all of these disorders are reversible on prompt and fitting intercession (4).

Erythropoietin (EPO) and its receptors are highly expressed in different body organs and tissues including kidneys and peripheral nerves at different levels. Also, on Schwann cells after peripheral nerve damage which might propose its gainful role in treating or even preventing any disorders affecting either the kidneys and or the nervous system (5).

Research hypothesis: in this study we hypothesized that EPO may have a protective effect on nerves in patients with renal failure either indirect via preventing kidney functions deterioration and subsequent nerve affection or through a direct protective effect on the nerves. So, we assumed that using EPO may be a doubleedged sword in renal patients.

Objective: this work had been carried out to conjecture any possible protective or curative impacts of EPO on the neuropathy accompanying acute renal damage in fully-grown male rats and the possible underlying mechanisms.

\section{MATERIALS AND METHODS}

\subsection{Animals}

This study had been conveyed out in strict accordance with the approved ethical guidelines, habitation, injections and electrophysiological studies all were carried out over one week in January, 2019, in the experimental animal unit in the Physiology Department; Faculty of Medicine; Cairo University, Egypt; after that the biochemical parameters had been measured in the Biochemistry Department; Faculty of Medicine; Ain Shams University, Egypt.

Forty fully-grown Wistar male rats weighing 180-230 $\mathrm{g}$ had been housed separately in wire mesh cages at normal; room temperature and light/dark cycle with relative humidity. Also, they had been left to adapt to this environment for 72 hours preceding the starting of the actual experimental procedure, during which they had been fed ad libitum and tap water.

\subsection{Experimental design}

Then afterward all rats had been starved of water for 24 hours then haphazardly separated into four groups preceding the injection of saline or medications. Group I (control): rats had been injected with isotonic saline $1.2 \mathrm{~g} / \mathrm{kg}$ i.p. Group II (ARF): rats had been injected with a single dose of amikacin (1.2g/kg i.p) (6), Group III (ARF + EPO): rats had been injected with a single dose of amikacin $(1.2 \mathrm{~g} / \mathrm{kg}$ i.p), 24- hours later they had been injected with a single dose of erythropoietin (2000IU/Kg, i.p). Group IV (EPO+ARF): rats had 
received a single dose of erythropoietin (2000IU/Kg, i.p) concomitant with a single dose of amikacin (1.2g/kg i.p) (7), All groups had been water deprived 24 hours before the start of injection (8).

\subsection{Chemicals}

Erythropoietin had been gotten from Sedico (Egypt) in the form of epoetin 4000 IU and amikacin had been gotten from BMS (Egypt) in the form of amikin $500 \mathrm{mg}$ vials. Chloroform had been purchased from Sigma-Tec pharmaceutical industries (Egypt).

\subsection{Urine collection}

Urine had been gathered over 24-hours, separately for each rat utilizing special cages as those depicted by Demirkan and Melli (9).

\subsection{Estimation of blood biochemical parameters}

24 hours after the last injection, the rats had been anesthetized with chloroform inhalation, (10) then retro-orbital blood samples had been withdrawn from all rats, (11) for subsequent determination of serum creatinine, BUN levels. Creatinine had been assessed by Quanti Chrom TM creatinine test kit (12). GFR had been measured by Creatinine clearance $=\mathrm{u} / \mathrm{p} \times \mathrm{v}$ where $\mathrm{u}=$ urinary concentration of creatinine $(\mathrm{mg} / 100 \mathrm{ml}) ; \mathrm{p}=$ plasma concentration of creatinine $(\mathrm{mg} / 100 \mathrm{ml}) ; \mathrm{v}=$ urine volume $(\mathrm{ml} / \mathrm{min})$. Serum urea had been estimated by Quanti Chrom TM urea assay kit (13). Then all rats had been sacrificed and the sciatic nerves had been dismembered.

\subsection{Estimation of sciatic nerve}

electrophysiological parameters

The sciatic nerve had been presented through a gluteal muscle incision then excised (14).

\section{Setup and calibration of equipment:}

1) The red and black alligator clips from the stimulator electrodes were connected to two of the metal rungs on opposite sides of the MLT012/B nerve bath. The distance between the electrodes was $0.5 \mathrm{~cm}$. It was not necessary to connect the green (ground) alligator clip. Because the CAP is initiated at or near the cathode, and the anodal action slows down or may even suppress the CAP, the cathode should always be closer to the recording electrodes.

2) The red (positive) connector from the stimulator electrode was connected to the positive $(+)$ analog output connector on the Power Lab. We connected the black (negative) connector from the stimulator electrode to the negative (-) analog output connector.

3) The red and black leads from the recording electrode were connected to two of the metal rungs of the MLT012/B nerve bath. We connected the 8pin pod connector to the Pod port on Input 2 of the Power Lab.

4) Using an eyedropper or Pasteur pipette, we filled the lower reservoir of the nerve bath with modified Ringer's solution. Fluid in the lower reservoir must not come in contact with the metal electrode rungs as overfilling the nerve bath in this manner will cause a short circuit in the experiment. 5) We cut a strip of filter paper and laid it over the wires in the nerve bath so that it touched both stimulating electrodes and both recording electrodes. We moistened the paper strip with modified Ringer's solution, and placed the cover on the nerve bath. This arrangement was used to test our connections.

6) The Power lab was turned on and was connected to the USB port on the computer.

7) Chart was launched from the computer. From the Experiments Gallery we located and doubleclicked on the file called CAP Set Chart.

8) We obtained the isolated rat sciatic nerve. A small slip of bone/muscle was left attached to the 
nerve at the proximal end. A piece of thread was tied to the nerve at the distal end. Using forceps we lifted the nerve out of its dish by grasping the thread tied to either end of the nerve (as grasping the nerve with forceps will damage the nerve).

9) Gently we blotted the nerve on a piece of tissue or filter paper to remove any excess modified Ringer's solution.

10) We removed the filter paper from the nerve bath. We laid the nerve across the wire electrodes'; making sure it was in contact with each of the active connections. The nerve was placed in the chamber with the bone end near the two stimulating electrodes (anode and cathode). The lid of the chamber was then placed firmly on top to preserve the humidified air in the bath. The stainless-steel electrodes in the bath were equally spaced.

Afterward via using the computer system; power lab and adjusting the stimulator as following: stimulus duration $(0.1 \mathrm{sec})$, voltage $(0.5$ v) at a frequency of $1 \mathrm{~Hz}$ in a repeated mode, 15 the NCV had been computed as follow: velocity = distance / latency in $(\mathrm{m} / \mathrm{sec}), 16$ and the compound action potential's amplitude had been noticed as in figure (1).

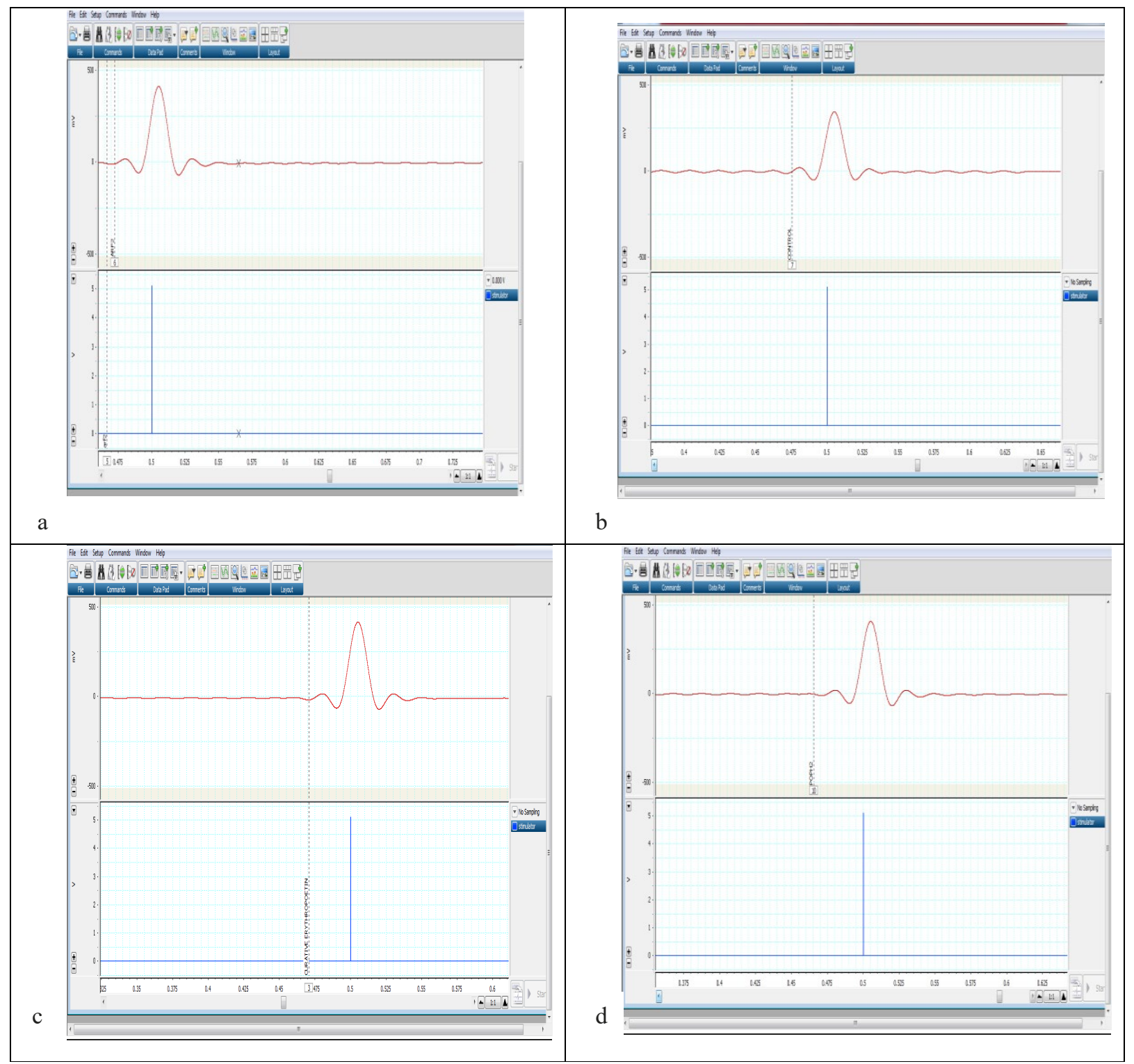

Figure (1): Shows recorded sciatic nerve action potential among the different studied groups via powerlab a) Group I, b) Group II, c) Group III, and d) Group IV. 
2.7 Estimation of MDA, SOD and NGF levels in nerve tissues

At a $\mathrm{pH}$ of 7 , the sciatic nerves - after weighing - had been rinsed in ice- cold saline solution and frozen in liquid nitrogen then they had been cut into small pieces, homogenized at $4^{\circ} \mathrm{C}$ in $2 \mathrm{ml}$ of ice-cold saline then filtered and used for the determination of MDA, superoxide dismutase and NGF according to the methods described by Knight (17), Nishikimi (18) and Weskamp and Otten (19) respectively.

-MDA was measured by BioVision's Lipid Peroxidation (MDA) Colorimetric/Fluorometric Assay Kit, Catalog \#: K739, United States. Kit Components: MDA Lysis Buffer, Phosphotungstic Acid Solution, BHT (100X) TBA and MDA Standard (4.17 M)

Principle: The MDA in the sample is reacted with Thiobarbituric Acid (TBA) to generate the MDATBA adduct. The MDA-TBA adduct can be easily quantified colorimetrically $(\lambda=532 \mathrm{~nm})$ or fluorometrically $(\mathrm{Ex} / \mathrm{Em}=532 / 553 \mathrm{~nm})$.

- SOD was measured by BioVision's rat SOD ELISA Kit, Cat.\#: MBS3807791, United States. Kit components: Microelisastripplate, Sample diluent, HRP-Conjugate reagent, Wash solution, Chromogen Solution, Stop Solution, Closure plate membrane and Sealed bags.

Principle: The Stop Solution changes the color from blue to yellow and the intensity of the color is measured at $450 \mathrm{~nm}$ using a spectrophotometer.

Table (1): Comparison between the mean levels of serum creatinine (mg/dl), BUN (mg/dl), 24-hours urine volume $(\mathrm{ml})$ and GFR (ml/min) among all studied groups

\begin{tabular}{lllll}
\hline & $\begin{array}{l}\text { Control } \\
\text { (Group I) }\end{array}$ & $\begin{array}{c}\text { ARF } \\
\text { (Group II) }\end{array}$ & $\begin{array}{l}\text { ARF+EPO } \\
\text { (Group III) }\end{array}$ & $\begin{array}{l}\text { EPO+ARF } \\
\text { (Group IV) }\end{array}$ \\
\hline Serum creatinine (mg/dl) & $0.45 \pm .05$ & $1.64 \pm .23^{\#}$ & $0.51 \pm 0.19^{@}$ & $0.78 \pm .07^{\# @ \$}$ \\
BUN (mg/dl) & $37.00 \pm 2.71$ & $68.99 \pm 3.70^{\#}$ & $42.94 \pm 2.69^{\# @}$ & $49.81 \pm 2.10^{\# @ \$}$ \\
24-hours urine volume (ml) & $6.15 \pm .68$ & $3.07 \pm .53^{\#}$ & $5.09 \pm 0.26^{\# @}$ & $4.48 \pm .29^{\# @ ~}$ \\
GFR (ml/min) & $1.64 \pm 0.15$ & $0.70 \pm 0.13^{\#}$ & $1.34 \pm 0.14^{\# @}$ & $1.08 \pm 0.14^{\# @ \$}$ \\
\hline
\end{tabular}

Values are represented as mean \pm SD. ${ }^{\#}$ statistically significant compared to its corresponding value in group (I) (P<0.05). ${ }^{\circledR}$ statistically significant compared to its corresponding value in group (II) $(\mathrm{P}<0.05)$. ${ }^{\$}$ statistically significant compared to its corresponding value in group (III) $(\mathbf{P}<0.05)$.BUN: blood urea nitrogen, GFR: glomerular filtration rate, ARF: acute renal failure, EPO: erythropoietin.
- $\beta$-NGF was measured by Rat $\beta$-NGF ELISA Kit \#RAB0381, Sigma Aldrich Chemie GmbH, Germany. Kit Components: 20X Wash Buffer, HRP-Streptavidin, ELISA Colorimetric TMB

Reagent (HRP Substrate, Item H), ELISA Stop Solution

ELISA $1 \mathrm{X}$ Assay/Sample, Diluent Buffer A (ELISA 5X Assay/Sample) and Diluent Buffer B.

\subsection{Statistical methods}

The measured variables - both chemical and electrical - had been analyzed and contrasted with their relatives in all groups by the product SPSS as means \pm SD and analysis of variance; post hoc test individually. P-values more than 0.05, had been considered as measurably insignificant (20).

\section{RESULTS}

3.1 Assessment of the kidney functions in all experimental groups

As shown in table (1), there had been a significant increase of both serum creatinine and blood urea nitrogen (BUN), and a significant decrease in 24-hours urine volume and GFR in ARF group relative to control group. But these parameters had been significantly corrected in both group III and group IV, although these measures had not attained yet the normal control values, except for the serum creatinine in group III. 
3.2 Assessment of the sciatic nerve conduction velocity (NCV) and amplitude in all experimental groups

As illustrated in table (2), the latent period had been significantly increased in ARF group and had been significantly decreased again in both group III and group IV. Consequently, the NCV had been significantly diminished in group II relative to group I, but these parameters had been significantly improved in rats that had been injected with EPO either before or with induction of ARF. In addition, the CAṔs amplitude had been significantly diminished in ARF group relative to control group and markedly enhanced in both $\mathrm{ARF}+\mathrm{EPO}$ and EPO+ARF groups. Despite these marked improvements in NCV and CAPs amplitude but they had not returned back to the normal control values.

Table (2): Comparison between the mean values of sciatic NCV (m/sec) and amplitude (mv) among all studied groups

\begin{tabular}{lcccc}
\hline & $\begin{array}{c}\text { Control } \\
\text { (Group I) }\end{array}$ & $\begin{array}{c}\text { ARF+EPO } \\
\text { (Group III) }\end{array}$ & $\begin{array}{c}\text { ARF } \\
\text { (Group II) }\end{array}$ & $\begin{array}{c}\text { EPO+ARF } \\
\text { (Group IV) }\end{array}$ \\
\hline Distance (m) & 0.005 & 0.005 & 0.005 & 0.005 \\
Latent period (sec) & $0.0043 \pm .0 .0001$ & $0.0071 \pm .0 .0001^{\#}$ & $0.0049 \pm .0 .0002 \# @$ & $0.0055 \pm .0 .006 \# @ \$$ \\
NCV (m/sec) & $1.16 \pm .06$ & $0.70 \pm .10^{\#}$ & $1.03 \pm .05^{\# @}$ & $0.91 \pm .10^{\# @ s}$ \\
CAP amplitude (mv) & $486.11 \pm 7.64$ & $376.92 \pm 27.93^{\#}$ & $455.74 \pm 21.91^{\# @}$ & $423.28 \pm 30.6^{\# @ s}$ \\
\hline
\end{tabular}

Values are represented as mean \pm SD.

${ }^{\#}$ statistically significant compared to its corresponding value in group (I) $(\mathrm{P}<0.05)$.

${ }^{\circledR}$ statistically significant compared to its corresponding value in group (II) $(\mathrm{P}<0.05)$.

$\$$ statistically significant compared to its corresponding value in group (III) $(\mathrm{P}<0.05)$.

NCV: nerve conduction velocity, CAP: compound action potential, ARF: acute renal failure, EPO: erythropoietin.

3.3 Assessment of malondialdehyde (MDA), superoxide dismutase (SOD) and nerve growth factor (NGF) levels in sciatic nerve in all experimental groups

Table (3) revealed a significant rise in MDA, and a significant decline in SOD and NGF levels in nerve tissues following induction of ARF relative to the control rats, the treatment with EPO in group III showed a significant improvement of these assessed parameters with nearly normalized MDA level. Unlike, no significant improvement in either MDA or SOD levels had been noticed on the prophylactic administration of EPO as in group IV, just a significant improvement of NGF level had been recorded.

Table (3): Mean levels of MDA (nmol/mg protein), SOD (U/mg protein) and NGF (pg/mg) among all studied groups

\begin{tabular}{lllll}
\hline & $\begin{array}{l}\text { Control } \\
\text { (Group I) }\end{array}$ & $\begin{array}{l}\text { ARF } \\
\text { (Group II) }\end{array}$ & $\begin{array}{l}\text { ARF+EPO } \\
\text { (Group III) }\end{array}$ & $\begin{array}{l}\text { EPO+ARF (Group } \\
\text { IV) }\end{array}$ \\
\hline MDA (nmol/mg protein) & $22.33 \pm 5.33$ & $30.97 \pm 2.67^{\#}$ & $25.39 \pm 3.93^{@}$ & $28.28 \pm 4.29^{\#}$ \\
SOD (U/mg protein) & $9.60 \pm 0.61$ & $5.62 \pm 0.84^{\#}$ & $7.84 \pm 0.54^{\# @}$ & $7.3360 \pm 1.80^{@ \$}$ \\
NGF (pg/mg protein) & $26.09 \pm 1.47$ & $13.56 \pm 0.59^{\#}$ & $20.98 \pm 0.98^{\# @}$ & $17.25 \pm 1.20^{\# @ \$}$ \\
\hline
\end{tabular}

Values are represented as mean \pm SD. ${ }^{*}$ statistically significant compared to its corresponding value in group (I) (P<0.05).

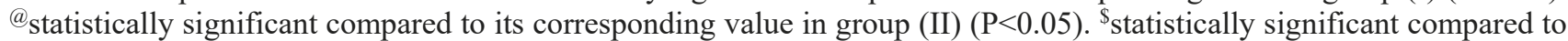
its corresponding value in group (III) $(\mathrm{P}<0.05)$. MDA: malondialdehyde, SOD: superoxide dismutase, NGF: nerve growth factor, ARF: acute renal failure, EPO: erythropoietin. 


\section{Discussion}

In the present study, the well-known nephrotoxic impact of amikacin on the kidneys; through bringing on intense renal tubular necrosis, besides renal vasoconstriction (21), had been manifested by a significant rise of serum creatinine as well BUN levels with a significant decrease in 24- hours urine volume and GFR in group II.

Moreover, nerve conduction studies were carried out to assess the functional status of one of the peripheral nerves (sciatic nerve) as these studies are very sensitive to changes in both neuronal axons and myelin sheath that might be associated with renal failure either by recording latency which is the time it takes to activate the nerve and calculating the $\mathrm{NCV}$ or recording the nerve amplitude CMAP which is the summation of individual motor unit action potentials, and it is indicative of axonal integrity.

Upon these studies, a lot of deleterious changes had been recorded in the sciatic nerve fibers accompanying induction of ARF as diminished: NCV, compound action potential's amplitude that mostly reflected axonal fibers loss, SOD and NGF, in addition to raised MDA levels in nerve tissues and latency that most probably revealed defect in nerve myelination. This deterioration of nerve functions might be clarified by diminished EPO secretion by the insulted kidneys with subsequent anemic hypoxia and ischaemia, therefore energy is no longer available for different body tissues including peripheral nerves (22).

Additionally, the nerves might be straightforwardly influenced by the retained neuroand or uremic toxins (23), besides, the electrolytes disturbances that typically go with ARF influencing basically $\mathrm{Na}+, \mathrm{K}+$ and $\mathrm{Ca}++$ concentrations that might disturb the nerve membrane potential and excitability with resultant diminished nerve conduction velocity (24).

Also, the developed metabolic acidosis is another factor predisposing to diminished nerve excitability (25). It had been obvious that the severe decline in both kidney and nerve functions in group II, had been improved upon treatment with EPO (group III) and had become less deteriorated on prophylactic intake of EPO which might reflect the beneficial effect of EPO on the speed and strength of nerve signals.

These beneficial impacts are mostly interceded by stimulating the JAK2-STAT5 pathway taking after EPO - EPORs binding, with resulting activation of many other signaling pathways (26), antagonizing apoptosis possibly through incitement of heat shock protein 70 (HSP70) (27) and XIAP (28) or inactivation of caspases (29) or GSK3 $\beta$ (30) and or through inhibiting FOXO3a (31) or stopping cytochrome c release (32). As apoptosis is an irreversible process, punctual intercession with a neuroprotective therapy as erythropoietin may be useful in diminishing its occurrence or even much reversing it (33). All these impacts, intercede both the erythropoietic and non-erythropoietic actions of EPO (34).

Moreover, EPO exerts an antiinflammatory impact via decreasing release of interleukin-2 and tumour necrosis factor, in ischemia reperfusion renal injury (35), and undo the effect of endotoxins on the antioxidant; renal SOD through NF-kB pathway and EPO enhances the expression of SOD via nitric oxide signaling pathway (36). EPO diminishes free radicals 
generation and lipid peroxidation and consequently influences the neurotransmitters release (37) as it had been confirmed in this work, by the decrease in MDA level.

Additionally, EPO has a protective impact on vasculature since it promotes neovascularization directly through upregulating vascular endothelial growth factors and their receptors 38 , and indirectly through promoting the movement of endothelial progenitor and bone marrow-derived proangiogenic cells (39). It had been reported that angiogenesis would be impaired in absence of erythropoietin receptors (40).

EPO injection either concomitant with (as in group IV) or following induction of ARF (as in group III), had attained a significant improvement in both the NCV and amplitude that may be owed to the crucial role of EPO in development, maintenance, protection and moreover repair of the nervous system (41). It had been reported that neuronal expression of EPO and its receptors is enhanced subsequent to peripheral nerve injury (41). As EPO -via $\beta 1$ integrin - plays a crucial role in proliferation and migration of Schwann cells to areas of neural injury (42).

Being a renal patient, receiving EPO, you will get the greater part of its profits whichever treating extreme anemia, recuperation of renal functions, correcting or attenuating complications, such that peripheral neuropathy.

Ultimately, we must weigh the benefits versus the adverse effects of EPO as a treatment option for renal patients or even neuropathic patients, particularly the hazard of thrombosis (43). Proper history taking, examination and investigation of the patients before prescribing it as a treatment are mandatory and must be done cautiously. Also strict follow up of patients on EPO is a must, as there is an increased incidence of hypertension due to more endothelin release, upregulation of tissue renin and imbalance of vasoactive substances (44).

Conclusion: this work emphasized the EPO neuroprotective impact in rats with acute renal shutdown. It showed that the curative effect of EPO is superior to its prophylactic one but both regimens scored a significant improvement.

Recommendation: this study has to be conducted in patients rather than animals trying different erythropoietin variants hoping to find an erythropoietin derivative with a non hematopoietic action as a treatment of different neurological dysfunctions to limit its adverse effects.

\section{ACKNOWLEDGEMENT}

We would like to express special thanks of gratitude to our department chairs that had provided us general support and to Mrs. Afaf for technical help.

FINANCIAL SUPPORT AND SPONSORSHIP Nil.

\section{CONFLICT OF INTEREST}

There had been no conflict of interest.

\section{ORCID}

http://orcid.org/0000-0002-3985-8931

\section{REFERENCES}

1. Jacob R. Acute renal failure. Indian J Anaesth. 2003; 47: 367-372.

2. Krishnan AV, Kiernan MC. Uremic neuropathy: clinical features and new 
pathophysiological insights. Muscle

Nerve. 2007; 35(3):273-90.

3. Ropper AH. Accelerated Neuropathy of Renal Failure. Arch Neurol. 1993;50 (5):536-539.

4. Ghazan-Shahi S, Koh T, Chan C. Impact of nocturnal hemodialysis on peripheral uremic neuropathy. BMC Nephrology; 2015:16(1):1

5. Bernaudin M, Bellail A, Marti H, Yvon A, Vivien D, Duchatelle I, Mackenzie ET, Petit E. Neurons and astrocytes express EPO mRNA: oxygen-sensing mechanisms that involve the redox-state of the brain". Glia. 2000; 30 (3): 271-8.

6. Kaynar K, Gul S, Ersoz S, Ozdemir F, Ulusoy H, Ulusoy S. Amikacin-Induced Nephropathy: Is There Any Protective Way?. Renal failure. 2007; 29. 23-7.

7. Kaynar K, Aliyazioglu R, Ersoz S, Ulusoy S, Al S, Ozkan G, Cansiz M. Role of erythropoietin in prevention of amikacininduced nephropathy. J Nephrol. 2012; 25(5):744-9.

8. Aydogdu N, Atmaca G, Yalcin O, Taskiran R, Tastekin E, Kaymak K. Protective effects of L-carnitine on myoglobinuric acute renal failure in rats. Clin Exp Pharmacol Physiol. 2006; 33(1-2):119-24.

9. Demirkan, A, Melli, M. A: simple and inexpensive device for collecting urine samples from rats. Lab Anim (NY). 2007; $36: 39-41$.

10. Moke G, Erhirhie E, Ahante E. Effects of inhaled anaesthetic agents (Chloroform and Diethyl ether) on fasting blood glucose and haematological parameters in Wistar rats. Sky Journal of Biochemistry Research. 2015; 4: 13-15
11. Parasuraman S, Raveendran R, Kesavan R. Blood sample collection in small laboratory animals. $\quad J \quad$ Pharmacol Pharmacother. 2010;1(2):87-93.

12. Bergman A, Ohman G. Effect of detergent on kinetic Jaffé-method assay of creatinine. Clin Chem. 1980; 26 (12):1729-32.

13. Jung $\mathbf{D}$, Biggs $\mathbf{H}$, Erikson $\mathbf{J}$ and Ledyard, P. U: New colorimetric reaction for end-point, continuous-flow, and kinetic measurement of urea. Clinical chemistry. 1975; 21: 1136.

14. Eliasson SG. Nerve conduction changes in experimental diabetes. J Clin Invest. 1964; 43: 2353-8.

15. Leal-Cardoso JH, Matos-Brito BG, LopesJunior J.E.G, Viana-Cardoso K.V, Sampaio-Freitas A.B, Brasil R.O, Coelhode-Souza A.N, Albuquerque A.A.C. Effects of estragole on the compound action potential of the rat sciatic nerve. Brazilian journal of medical and biological research. 2004; 37(8): 1193- 8 .

16. Hunter.I.W, Kearney R. E, Jones L. A. Estimation of the conduction velocity of muscle action potentials using phase and impulse response function techniques. Medical and biological engineering and computing. 1987;25(2):121.

17. Knight J. A, Pieper R. K, Mc Clellan L. Specificity of the thiobarbituric acid reaction:its use in studies of lipid peroxidation. Clin. Chem. 1988; 34: 2433. Nishikimi, M, Roa N.A, Yogi. K. The occurrence of superoxide anion in the reaction of reduced phenazine methosulfate and molecular oxygen. Biochem. biophys. res. commun. 1972; 46: 849-854. 
18. Weskamp G, Otten U. An enzyme-linked immunoassay for nerve growth factor (NGF): a tool for studying regulatory mechanisms involved in NGF production in brain and in peripheral tissues. J. Neurochem. 1987; 48: 1779-1786.

19. Chan Y.H. Biostatistics102: Quantitative data - parametric \& non-parametric tests. Singapore Med J. 2003; 44(8): 391-6.

20. Lopez-Novoa J.M, Quiros Y, Vicente L, Morales A, Lopez-Hernandez F. New insights into the mechanism of aminoglycoside nephrotoxicity: an integrative point of view. Kidney International. 2011; 79(1): 33-45.

21. Işıkay S, Işııkay N, Sarsu SB, Belen FB. Peripheral neuropathy: not a feature of childhood thalassemia. Ann Med Health Sci Res; 2017; 7:1-4.

22. Said G. Uremic neuropathy. HandbClin Neurol. 2013; 115:607-12.

23. Keshavan A, Gandhi S, Lunn M, Reilly MM. Electrolyte imbalance triggering relapse of inflammatory neuropathy. $\mathrm{j}$ neurolneurosurg psychiatry. 2013;84:e2.

24. Aramideh, M, Koopmans R P, Koelman, J.H, Stam J. Rapidly progressing peripheral neuropathy with lactic acidosis and coproporphyria in a patient with HTLV-I associated T-cell leukaemia treated with zidovudine. Deutsche Zeitschriftfür Nervenheilkunde. Journal of Neurology. 2001;248(7): 621-622.

25. Breggia AC, Wojchowski DM, Himmelfarb J. JAK2/Y343/STAT5 signaling axis is required for erythropoietin-mediated protection against ischemic injury in primary renal tubular epithelial cells. Am J Physiol Renal Physiol. 2008; 295(6): 689-95.

\section{Xu B, Dong GH, Liu H, Wang YQ, Wu} HW, Jing H. Recombinant human erythropoietin pretreatment attenuates myocardial infarct size: a possible mechanism involves heat shock protein 70 and attenuation of nuclear factor-kappa B. Ann Clin Lab Sci. 2005 Spring; 35(2):161-8.

27. Digicaylioglu M, Garden G,Timberlake S, Fletcher L, Stuart A. Acute neuroprotective synergy of erythropoietin and insulin-like growth factor I. Proceedings of the National Academy of Sciences. 2004; 101 (26): 9855- 860.

28. Yang B, Hosgood SA, Bagul A, Waller HL, Nicholson ML. Erythropoietin regulates apoptosis, inflammation and tissue remodelling via caspase-3 and IL-1 $\beta$ in isolated hemoperfused kidneys. Eur J Pharmacol. 2011; 25(660):2-3.

29. Inkster B, Zai G, Lewis G, Miskowiak K: GSK3ß: a plausible mechanism of cognitive and hippocampal changes induced by erythropoietin treatment in mood disorders? Translational Psychiatryin mood disorders.2018; 8(216).

30. Maiese K: "Regeneration in the nervous system with erythropoietin,". Frontiers In Bioscience-Landmark. 2016;21:561-596.

31. Ifeanyi O. E. A Review on Erythropoietin. Int. J. Adv. Res. Biol.Sci. 2015; 2(4):35-47.

32. Ghezzi P, Brines M. Erythropoietin as an antiapoptotic, tissue-protective cytokine. Cell Death Differ. 2004; 11Suppl 1:S37-44.

33.

Brines M, Patel N.S.A,Villa P, Brines 
C, Mennini T, De Paola M, Erbayraktar

Z, Erbayraktar

S, Sepodes

B, Thiemermann

C, Ghezzi

P, Yamin

M, Hand C, Xie Q, Coleman T, Cerami A.

Nonerythropoietic, tissue-protective peptides derived from the tertiary structure of erythropoietin. Proceedings of the National Academy of Sciences. 2008; 105 (31): 1092510930.

34. Bartnicki P, Kowalczyk M, Rysz J. The influence of the pleiotropic action of erythropoietin and its derivatives on nephroprotection. Med SciMonit. 2013; 19:599-605.

35. Mitra A, Bansal S, Wang W, Falk S, Zolty E and Schrier R.W: Erythropoietin ameliorates renal dysfunction during endotoxemia. Nephrology, dialysis, transplantation: official publication of the European Dialysis and Transplant Association - European Renal Association; 2007, 22. 234953. 10.

36. Hassan A, Abd El-Rahim A: Management by Erythropoietin and Nerve Growth Factor on Healing of Spinal Cord Injury in Rats. International Journal of Biology. 2013;5(2):111.

37. Kimáková $\mathbf{P}$, Solár $\mathbf{P}$, Solárová $\mathbf{Z}$, Komel $\mathbf{R}$ and Debeljak N. Erythropoietin and Its Angiogenic Activity. Int J Mol Sci. 2017; 18(7):1519.

38. Ostrowski D and Heinrich R: Alternative Erythropoietin Receptors in the Nervous System. Journal of Clinical Medicine.2018; 7(2): 24.
39. Nakano M, Satoh K, Fukumoto Y, Ito Y, Kagaya Y, Ishii N, Sugamura K, Shimokawa H: Important role of erythropoietin receptor to promote VEGF expression and angiogenesis in peripheral ischemia in mice. Circulation Research. 2007;100:662-669.

40. Wang W, Li D, Li Q, Wang L, Bai G, Yang T, Li Q, Zhu Z, Sun H. Erythropoietin promotes peripheral nerve regeneration in rats by upregulating expression of insulin-like growth factor-1. Arch Med Sci. 2015; 11(2):433-7.

41. Zhang W, Gao Y, Zhou Y, Liu J, Zhang L, Long A, Zhang L, Tang P: Localized and sustained delivery of erythropoietin from PLGA microspheres promotes functional recovery and nerve regeneration in peripheral nerve injury. Biomed Res Int. 2015:478103.

42. Chavez-MacGregor M, Zhao H, Fang S, Srokowski TP, Hortobagyi GN, Giordano SH. Complications associated with erythropoietin-stimulating agents in patients with metastatic breast cancer: a surveillance, epidemiology, and end results-medicare study. Cancer. 2011; 117(16):3641-9.

43. Aachmann-Andersen N. J, Christensen S. J, Lisbjerg K, Oturai P., Johansson P. I, Holstein-Rathlou N.-H, Olsen N. $\mathbf{V}$ : Recombinant erythropoietin acutely decreases renal perfusion and decouples the rennin- angiotensin- aldosterone system. Physiol Rep. 2018; 6 (5): e13573. 